\title{
Our New Choice in Labour Analgesia, Combined Spinal-Epidural Analgesia: Our Short Term Results
}

\author{
Ahmet Gültekin ${ }^{1}$, Ebru Çanakçı ${ }^{2}$ \\ ${ }^{1}$ Namık Kemal University, Medical School, Department of Anesthesiology and Reanimation, Tekirdağ, Turkey \\ ${ }^{2}$ Ordu University, Medical School, Training and Research Hospital, Department of Anesthesiology and Reanimation, Ordu, Turkey \\ Received: 18 February 2020, Accepted: 12 April 2020, Published online: 30 April 2020 \\ (C) Ordu University Institute of Health Sciences, Turkey, 2020
}

\begin{abstract}
Objective: Nowadays, the painless childbirth preference of expectant mothers is gradually increasing. The most important and first objective in labor analgesia should be the safety of mother and baby. Our objective in this study is to compare the effects of combined spinal-epidural analgesia (CSE) and epidural analgesia on analgesia quality and labor in labour analgesia.
\end{abstract}

Methods: Our study was performed prospectively on 40 primigravid pregnant women who requested painless childbirth and were included in the ASAI-II group, between September 1, 2018, and November 30, 2018. Pregnant women who had single fetus at $36-42$ weeks, have vertex presentation, no contraindications for regional analgesia, have active contractions and with 3-4 cm cervical opening were randomly divided into two groups in the study. Combined Spino Epidural group (= Group CSE, $n=20$ ), Epidural analgesia group (= Group EPI, $\mathrm{n}=20$ ) was determined as the groups. To the pregnant women in the Group CSE, Intrathecal dose in a manner that $2.5 \mathrm{mg}$ hyperbaric bupivacaine $+12.5 \mu \mathrm{g}$ fentanyl total volume of $1 \mathrm{ml}$ was injected. To the pregnant women in the Group EPI, $20 \mathrm{G}$ epidural catheter was placed with the method used in the first group and fixed to the skin. Epidural analgesia solutions $(20 \mathrm{mg}$ bupivacaine $+50 \mu \mathrm{g}$ fentanyl $+15 \mathrm{cc}$ saline) were prepared in $20 \mathrm{cc}$ injectors containing $0.1 \%$ bupivacaine $+2.5 \mu \mathrm{g} / \mathrm{ml}$ fentanyl for use in both groups. When the VAS values became 4, these prepared solutions were planned to be given as $10 \mathrm{ml}$ intermittent bolus from these epidural catheters.

Results: The mean values of maternal age, weight, and height, and infant height have not shown any difference according to the groups ( $p>0.05$ ). The first VAS median value was obtained as 5 in both groups. However, the VAS values were accumulated in the higher values in the combined spinal-epidural group ( $\mathrm{p}$ $=0.031$ ). Although the second VRS and VAS median values were lower in the pure epidural, the outlet VRS and VAS values have been obtained lower in the combined spinal-epidural group.

Conclusion: For normal spontaneous vaginal delivery, every mother should be offered labour analgesia options such as CSE analgesia and epidural analgesia. CSE analgesia may be a good alternative to epidural analgesia because of its advantages such as the onset of fast analgesia, shortening the first stage of labour and increasing the comfort of the mother in the outlet.

Key words: painless childbirth, combined spinal-epidural analgesia, epidural analgesia

Suggested Citation: Gultekin A, Canakci E,. Our New Choice in Labour Analgesia, Combined Spinal-Epidural Analgesia: Our Short Term Results. Middle Black Sea Journal of Health Science, 2020; 6(1):99-105 


\section{Address for correspondence/reprints:}

Ebru Çanakçı

Telephone number: +90 5322651687

ORCID-ID 0000-0003-2093-9229

E-mail: canakciebru@gmail.com

DOI: $10.19127 / \mathrm{mbsjohs.690840}$

\section{Introduction}

Nowadays, the painless childbirth preference of expectant mothers is gradually increasing. However, the desire to have the mother to be given comfortable and painless childbirth should never endanger the safety of the baby and the mother (Thomson et al., 2019). The most important and first objective in labor analgesia should be the safety of mother and baby. The second objective should be the prevention of epidural or subarachnoid local anesthetic application related motor block; in other words, muscle weakness in the legs of the mother (Beilin et al.,2002).

The most effective way to eliminate the pain of labour is the central regional blocks. Epidural analgesia technique used in the relief of labour pain is the most widely accepted technique today due to providing continuous analgesia compared to other techniques, requiring lower concentrations of local anesthetics and analgesics, and not so much affecting the second-period expulsion movements by keeping motor loss to a minimum (Glosten et al.,2000 ) In our clinic, epidural analgesia is more preferred in labor analgesia. CSEA analgesia is our new choice.

The combined spinal-epidural (CSE) technique is becoming increasingly important in recent years because of the rapid onset of the analgesic effect and excellent analgesia without a significant reduction in motor functions. The reliability and efficacy of labour analgesia formed by this method have been shown in several studies (Gary et al.,2001; Erdine et al.,2005).

Our objective in this study is to compare the effects of combined spinal-epidural analgesia (CSE) and epidural analgesia on analgesia quality and labor in labour analgesia.

\section{Methods}

Ethical approval for our study was obtained from Ordu University Clinical Research Ethics Committee. (Decision no: 2018/245, Date: 29.11.2018). Our study was performed prospectively, unblinded on 40 primigravid pregnant women who requested painless childbirth and were included in the ASAI-II group, between September 1, 2018, and November 30, 2018. Randomization was performed according to the preference of the pregnants. The volunteering approval form has been signed by the pregnant women included in the study. Pregnant women who had single fetus at 36-42 weeks, have vertex presentation, no contraindications for regional analgesia, have active contractions and with $3-4 \mathrm{~cm}$ cervical opening were randomly divided into two groups in the study. Combined Spino Epidural group (= Group CSE, $\mathrm{n}=20$ ), Epidural analgesia group (= Group EPI, $n=20$ ) was determined as the groups. Pregnant women with severe neurological disease, preeclampsia, diabetes mellitus, infection at the insertion site, bleeding and coagulation disorder, who had known to have a susceptibility to amide-type local anesthetics, show excessive agitation and could not be cooperated were not included in the study. Pregnant women were

given information about the "Visual Analog Scale" (= VAS) which they will evaluate their pain during labour $(0 \mathrm{~cm}=$ no pain, $10 \mathrm{~cm}=$ the most severe pain). The cases were also informed about the VRS (= Verbal Rating Scale). They were told that 0 : no pain, 1: mild pain, 2: irritating pain, 3: moderate pain, 4: severe pain, 5: very severe pain. VAS and VRS scores were recorded at the time of the implementation of the first and second epidural analgesia doses. Our researcher fellows and clinician doctors followed the cases. Before starting the analgesia, the pregnant women were established vascular access with $18 \mathrm{G}$ intravenous cannula on the forearm, and $10 \mathrm{ml} / \mathrm{kg} / \mathrm{h}$ Ringer Lactate solution was started to be given. It was planned to start giving analgesia to the cases when cervical dilatation was 4 $\mathrm{cm}$, and VAS was 4 or higher. Systolic and diastolic arterial pressures, heart rate and VAS and VRS values of the pregnant women were measured and recorded before the starting of the procedure. Uterus contractions and fetal heart sounds were monitored by cardiotocograph monitor. After all pregnant women were provided skin disinfection in sitting position with an appropriate antiseptic solution, $40 \mathrm{mg}$ of lidocaine (Jetmonal 2\% ampul, Adeka İlaç, Turkey) infiltration was performed from the L3-L4 or L4- L5 spaces subcutaneously and to the skin.

Epidural space was reached by using the loss of resistance technique on the midline with $18 \mathrm{G}$ Touhy needle (BBraun Combined Spinal / Epidural AG, Germany) in the pregnant women included in the Group CSE. Forty mg of $2 \%$ lidocaine containing 3 $\mathrm{ml}$ of test dose was administered through the catheter. 
After this procedure, we waited for 5 minutes and the procedure was resumed upon not observing motor block in the patient.

Then, with $27 \mathrm{G}, 136.5 \mathrm{~mm}$ spinal needle, subarachnoid space was reached by needle through needle technique, and after the clear CSF flow is seen, the intrathecal dose was injected in a manner that 2.5 mg hyperbaric bupivacaine $+12.5 \mu \mathrm{g}$ fentanyl with a total volume of $1 \mathrm{ml}$. Immediately following the removal of the spinal needle, the $20 \mathrm{G}$ epidural catheter was guided through a Touhy needle into the cephal. Once it was seen that no blood and CSF at the epidural catheter, the catheter tip was placed in a way that the $5 \mathrm{~cm}$ of the catheter tip to be in the epidural region, by making the centimeter calculation, and fixed. Similarly, in the pregnant women included in the Group EPI, epidural space was reached by using the loss of resistance technique on the midline with the $18 \mathrm{G}$ Touhy epidural needle (Perifix, BBraun Melsungen AG, Germany), and $20 \mathrm{G}$ epidural catheter was placed with the technique used in the first group and fixed to the skin. Epidural catheters were placed in the painless period between two contractions in all cases. Epidural analgesia solutions (20 mg bupivacaine $+50 \mu \mathrm{g}$ fentanyl $+15 \mathrm{cc}$ saline) were prepared in $20 \mathrm{cc}$ injectors containing $0.1 \%$ bupivacaine $+2.5 \mu \mathrm{g} / \mathrm{ml}$ fentanyl for being used in both groups. When the VAS values became 4, these prepared solutions were planned to be given as $10 \mathrm{ml}$ intermittent bolus from these epidural catheters. After epidural catheterization procedure, the pregnant women were placed in the left side position in order to prevent vena cava compression of the uterus. Hemodynamic values at this stage were recorded. A $20 \%$ decrease in blood pressure or $90 \mathrm{~mm} / \mathrm{Hg}$ of systolic arterial pressure was considered as hypotension, and it was planned to administer ephedrine $5 \mathrm{mg} / \mathrm{ml}$ intravenously if necessary. The pain scores of the pregnant women were recorded as VAS 1, VRS 1 at the time of the administration of the first dose from the epidural catheter, as VAS 2, VRS 2 at the time at which the second dose was administered, and as VAS end, VRS end at the time of suturing the episiotomy. After catheter removal, patients were asked for their satisfaction. The patient's sensory block was evaluated using the widely used Pinprick test $(0=$ normal sensation, $2=$ blinded sensation, $3=$ no senses) and the motor block was evaluated using the modified Bromage Scale ( 0 $=$ can straighten both legs, $1=$ do not have enough power to straighten the leg, $2=$ able to move only the knee , 3 = able to move only the foot). Sensory and motor blocks were evaluated at 5th, 10th, 15th, 30th, 45th, 60th minutes, and then every 30 minutes until full cervical dilatation was reached. The amount of the first dose of labor analgesia (how many $\mathrm{ml}$ ), time of second dose (how long after the first dose) and the total labor duration were recorded separately for each case. The VAS and VRS scores of the cases were recorded when the first dose and second dose were administered through the epidural catheter. The height, weight and head circumference of each newborn infant were recorded separately. VAS: Marked scale on 10-point line was used. The left side was referred to having no pain ( 0 point) and the right side was referred to unbearable pain (10 points). The patient was asked to show her pain on this line. Effective analgesia was considered to be provided in those with the VAS score of 4 and less. The levels of sensorial block were determined with the "pinprick" (pricking with the tip of needle) method. Sensory block was considered to develop in the lower extremities when numbness and hypoalgesia were felt between the dermatomes of L1 and S5. The cases were informed also about VRS. It was expressed as 0 : no pain, 1: mild pain, 2: disturbing pain, 3: moderate pain 4: severe pain 5: very severe pain. The VAS and VRS scores were recorded when the first and second dose of epidural analgesia were administered. The time for cervix to open from $3-4 \mathrm{~cm}$ to $10 \mathrm{~cm}$ was recorded as the 1st stage, and the time from the full opening to the birth of the baby was recorded as the 2nd stage. Patients that were to undergo cesarean section were administered $15 \mathrm{ml}(2 \%)$ lidocaine and $5 \mathrm{ml}(0.5 \%)$ bupivacaine through epidural catheter and were taken to the operation. The patients were asked about their satisfaction level after the removal of the catheter. Satisfaction level was evaluated as poor, moderate and good.

\section{Statistical Analysis}

The data were analyzed with the IBM SPSS v23 (IBM Corp., Armonk, NY, USA). Compliance with normal distribution was examined with ShapiroWilk. The unpaired t-test was used to compare the parameters showing normal distribution according to the groups, while the Mann Whitney -U test was used for non-normally distributed ones. The Friedman test was used for the change in time of in-group VRS and VAS values. Normally distributed data were presented as mean \pm standard deviation, while nonnormally distributed data were presented as median (min-max). The significance level was accepted as $\mathrm{p}<0.05$. 


\section{Results}

The comparisons of our groups with respect to quantitative data are presented in Table 1.

The mean values of maternal age, weight, and height, and infant height have not shown any difference according to the groups $(\mathrm{p}=$ $0.193,0.322,0.777,0.931$ respectively). The median values of infant height did not show any difference according to the groups $(p=0.066)$. The infant head circumference median value was obtained as 35.5 in those with a pure epidural, while as 35 in those with combined spinal-epidural. The median value of the first opening was obtained similar in both groups. However, a significant difference was observed in the group with pure epidural due to accumulation at the higher places $(\mathrm{p}=0.021)$. The second opening median value was obtained higher in the combined group.

Table 1: Comparisons of quantitative data by groups

\begin{tabular}{lccc} 
& $\begin{array}{c}\text { Group Epidural } \\
(\text { Group EPI }) \\
(\mathrm{n}=20)\end{array}$ & $\begin{array}{c}\text { Group Combined Spino } \\
\text { Epidural (Group CSE) } \\
(\mathrm{n}=20)\end{array}$ & $\mathrm{p}$ \\
\hline Maternal Age* & $24.0 \pm 4.0$ & $25.6 \pm 4.9$ & 0.193 \\
Maternal weight $* *$ & $72.7 \pm 10.6$ & $75.8 \pm 13.2$ & 0.322 \\
Maternal height $* *$ & $160.2 \pm 5.0$ & $160.6 \pm 6.2$ & 0.777 \\
Infant weight** & $3268.5 \pm 474.4$ & $3257.5 \pm 646.9$ & 0.931 \\
Infant height* & $50(47-51)$ & $50(47-53)$ & 0.066 \\
Infant head circumference* & $35.5(33-50)$ & $35(33-38)$ & $\mathbf{0 . 0 0 5}$ \\
The first cervical dilatation** & $5(4-6)$ & $5(4-7)$ & $\mathbf{0 . 0 2 1}$ \\
The second cervical dilatation $*$ & $(0-10)$ & $7(5-10)$ & $\mathbf{0 . 0 0 2}$ \\
The first VRS * & $2(1-6)$ & $2(1-3)$ & 0.533 \\
The first VAS * & $5(2-7)$ & $5(3-9)$ & $\mathbf{0 . 0 3 1}$ \\
The second VRS * & $0(0-4)$ & $1(1-2)$ & $\mathbf{0 . 0 0 1}$ \\
The second VAS * & $0(0-7)$ & $3(1-4)$ & $\mathbf{0 . 0 1 0}$ \\
VRS end $*$ & $1(0-2)$ & $0(0-1)$ & $<\mathbf{0 . 0 0 1}$ \\
VAS end & $2(0-4)$ & $0(0-1)$ & $<\mathbf{0 . 0 0 1}$ \\
\hline
\end{tabular}

* Mann Whitney U test, ** Unpaired t-test

VAS: Visual Analog Scale VRS: Verbal Rating Scale

The first VRS median values did not show any difference according to the groups $(p=0.533)$. The first VAS median value was obtained as 5 in both groups. However, the VAS values were accumulated in the higher values in the combined spinal-epidural group $(\mathrm{p}=0.031)$. Although the second VRS and VAS median values were lower in the pure epidural, the VRS end and VAS end values have been obtained lower in the combined spinal-epidural group.

When the first, second and outlet VRS values were compared in the groups themselves, the first VRS median value was obtained higher than the second and the outlet in the pure epidural group. There is no difference between the second and outlet median values. In the combined spinal-epidural application, while there was no difference between the first and second VAS values, the VAS end value was obtained lower.

In the VAS median value, there was no difference between the VAS end and the second values in the pure epidural group and the first VAS value was obtained higher than the others. In the combined spinal-epidural group there is a difference between the whole times. We did not have a case taken to Csection. Labor analgesia shortened the second stage of labor. All of our cases were satisfied with labor analgesia. All patients satisfaction levels are good. None of our cases had postspinal headache.

\section{Discussion}

Our pain scores were very low in the combined spinal-epidural group compared to the epidural analgesia group. We found that labour pain ended faster in the CSE group, and our VAS end and VRS end scores were significantly lower in the CSE group compared to the epidural analgesia group. The infant head circumference was found to be higher in the CSE group and was statistically significant. This suggests that infants with larger head circumference may be delivered without the need for intervention or caesarean section delivery with CSE analgesia. In addition, the fact that the higher cervical opening values measured at the time of the second dose were higher in the CSE group, suggests that CSE analgesia shortened the first stage of labour and accelerated cervical effacement and dilatation. It can be said that 
CSE analgesia is a good choice due to its advantages such as providing more active, effective analgesia in labour analgesia, shortening the first stage of delivery and facilitating the suturing of episiotomy without the need for additional analgesia.

CSE, which is used to provide rapid labour analgesia induction with minimal side effects, is a technique in which analgesia maintenance is provided with infusion by the epidural catheter placed after spinal analgesic medications. In a study comparing epidural analgesia with CSE analgesia, no difference was found in terms of normal vaginal, operative vaginal or caesarean section delivery, APGAR scores, umbilical artery blood gases, anesthesia complications and success percentages (Wong et al., 2009; Chestnut et al., 2009). The starting of the complete analgesia is significantly faster in CSE than the epidural. In particular, sacral analgesia starts very quickly compared to the lumbar epidural. This situation is very advantageous for pregnant women who want analgesia in the late period of the first stage of labour or whose labour is advancing fast. Since the doses required for spinal analgesia are much less than those of the epidural, the risk of systemic toxicity is low. Because of the less systemic absorption of spinal anaesthetic medications into the maternal circulation, the fetal plasma drug concentrations are even lower than the epidural. This results in less drug delivery to the mother and baby, which makes CSE analgesia superior to epidural analgesia (Wong et al., 2009; Chestnut et al., 2009). The risk of motor block and hypotension is eliminated by avoiding the sympathetic block with performed spinal analgesia by administering only opioid lipid-soluble drugs (Sezer AO et al., 2007; Wong CA et al., 2009; Chestnut DH et al., 2009; Gunaydin B et al., 2010). This method not only enables the mobilization of pregnant women but also provides ideal complete analgesia in pregnant women with stenotic heart disease. In addition, sometimes the catheter may not work in lumbar epidural analgesia. If the CSE technique is chosen from the beginning, the failure rate may be less. Nevertheless, CSE has some undesirable side effects. Although the risk of post dural puncture headache (PDPH) with fine-tipped spinal needles does not increase, a dural puncture is required in CSE to start analgesia. Another concern is the risk of postpartum neuraxial infection, which is rarely seen after dural puncture. Intrathecal opioid application induced pruritus is higher than the epidural. In addition, whether the epidural catheter is in place cannot be clearly understood until 1-2 hours after the start of analgesia. Therefore, CSE is not preferred in pregnant women in whom a functional epidural catheter is mandatory, difficult airway expected or with the risk of fetal heart rate (FHR) anomalies. CSE analgesia is frequently started by using the needle-through-needle technique on the midline in the lumbar region, and analgesia is maintained from the epidural catheter (Wong et al., 2009; Chestnut et al., 2009).In our study, no postspinal headache complications were encountered in none of the pregnant women to whom we applied CSE.

In another randomized controlled trial on the comparison of epidural analgesia and CSE neuraxial labour analgesia, it was shown that the ratios of labour and caesarean section were similarly affected (Sezer et al., 2007). In our study, none of the pregnant women in both groups had received intervention and taken into the caesarean section. Considering the effects of neuraxial analgesia only on the course of labour, the start of analgesia was shown to be 2-8 minutes with CSE, while it was 15-20 minutes with epidural (Wong et al., 2009).In our study, the start of analgesia in the CSE group was in a very short period of 2-3 minutes. In Group EPI, analgesia was started in 15-20 minutes as in the study of Wang et al. Our study results are compatible one-to-one with the literature. However, CSE analgesia, compared to epidural analgesia, has been shown to increase the rate of cervical dilatation and accelerate the labour, on the other hand, there is no difference between the two techniques (Wong et al., 2009; Chestnut et al., 2009). In spite of all these, the opinion of there is no need to be concerned on this issue is widely accepted in the world today. Because, the fact that women who complain more about pain during labour demand more analgesia, and thus the rate of delivery by caesarean section is higher, can be ranked among other factors of not progressing of labour. Fetal macrosomia, malposition (e.g., occiput posterior) and non-functional labour are painful conditions with high rates of operative labour such as forceps and caesarean section (Wong et al.,2009). In our study, we observed that in group CSE, cervical dilatation accelerated compared to the group EPI, and shortened the active phase of the first stage of labour, i.e., accelerated cervical dilatation.

The combined spinal-epidural technique has become increasingly important in labour analgesia in recent years due to the rapid start of the analgesic effect and providing excellent analgesia without significant reduction in motor functions (Olmez et al.,2003). In addition to the rapid start of analgesia, combined spinal-epidural analgesia (CSE) technique may prevent several problems in conventional epidural applications such as incomplete block, motor block, and weak sacral involvement. The epidural 
component plays an important role in the continuation of analgesia if the pregnant woman does not give birth in the duration of the action of intrathecal agents after the application of CSE analgesia (Bilgin et al., 2007). In previous studies, it was reported that especially most of the pregnant women who underwent the combined spinal-epidural technique in the advanced stages of fast-progressing labours gave birth at the spinal component of the technique without requiring epidural infusion (Landau et al.,2002; Apiliogulları et al.,2010; Poma et al.,2018; Braga et al.,2019). Since our study was performed on primigravid pregnant women, the number of our cases that gave birth at the spinal component of the neuraxial analgesia was low. However, in the CSE group, we determined that the first stage of labour was significantly shorter compared to the epidural analgesia group. CSE analgesia has been accelerating cervical dilatation. Our results are consistent with the literature.

In a recent 124-series study, Stocks et al. have found that the duration of analgesia duration was 56 min, $68 \mathrm{~min}$, and $77 \mathrm{~min}$, respectively, in their study of adding $5 \mu \mathrm{g}, 15 \mu \mathrm{g}$ and $25 \mu \mathrm{g}$ fentanyl in $2.5 \mathrm{mg}$ bupivacaine in CSE analgesia. They have detected that as the dose of intrathecal fentanyl increased, the duration of pruritus and analgesia increased, and lowdose fentanyl was found to be similar with high doses in providing fast and effective analgesia, however, the analgesic effect was shortened (Stocks et al.,2001). In our study, we added $12.5 \mathrm{mcg}$ fentanyl to $2.5 \mathrm{mg}$ bupivacaine, as well. We did not have a case with the complication of pruritus. Our results are similar to the literature results. In addition, our dose of intrathecal fentanyl is convenient with literature.

\section{Study limitations}

There are certain limitations in our study. The low number of cases is the first limiting factor. Due to the fact that our study was conducted in limited time, the fact that we could not examine the maternal and neonatal effects of CSE analgesia is the second limiting factor.

\section{Conclusion}

As a result, both the ASA (American Society of Anesthesiology) and the ACOG (American College of Obstetrics and Gynecology) have the opinion that being in pain of a person under the control of a doctor is not acceptable. For this reason, labour analgesia options such as CSE analgesia, epidural analgesia should be offered to each mother for labor. CSE analgesia may be a good alternative to epidural analgesia because of its advantages such as the rapid analgesia, shortening the first stage of labour and increasing the comfort of the mother at the episiotomy suturing. We believe that our study will shed light on long-termed, painless childbirth studies with large populations to be conducted in the future.

Ethics Committee Approval: Ethics committee approval was received for this study from Clinical Research Ethics Committee of Ordu University. Peer-review: Externally peer-reviewed.

Author Contributions: Concept- EÇ.; Design-E.Ç, AG.; Materials- E.Ç, AG.; Data Collection and/or Processing- E.Ç, AG. Literature Review- E.Ç, AG., E.Ç, AG.; Writing- E.Ç, AG.; Critical Review- E.Ç, AG.

Conflict of Interest: No conflict of interest was declared by the authors.

Financial Disclosure: The authors declared that this study hasn't received no financial support.

\section{References}

Apiliogulları S, Gok F, Kilicaslan A, Aydogan A, Celik JB. Our Experinces in Single-Shot Spinal Block for Labour Analgesia Selcuk Med J 2010;26(2):38-41.

Beilin Y. Advances in labor analgesia. Mt Sinai J Med 2002;69:38-44.

Bilgin F. Combined Spinal-Epidural Analgesia in Pain-Free Delivery Turkey Clinics J Surg Med Sci 2007;3:34-40.

Braga AFA, Carvalho VH, Braga FSDS, Pereira RIC. Combined spinal-epidural block for labor analgesia. Comparative study with continuous epidural block. Rev Bras Anestesiol. 2019; 69(1):7-12.

Erdine S. Obstetric anesthesia and analgesia applications. Regional Anesthesia. Nobel Medical Bookstore, İstanbul 2005:253-70.

Gary MS, Stephen PH. Minimum local analgesic dose of intrathecal bupivacaine in labor and the effect of intrathecal fentanyl. Anesthesiology 2001;94: 593-8.

Glosten B.Anesthesia for obstetrics. Miller RD (ed), In: Anesthesia, Churchill Livingstone, San Francisco. 2000; 2024-68.

Gunaydin B. Obstetric Pain and Its Management in the Perinatal Period: What do we need to know? In: Kuczkowski KM, Drobnik L (eds). International Textbook of ObstetricAnaesthesia and Perinatal Medicine. Medmedia, Warsaw 2010; 34-9.

Landau R. Combined spinal-epidural analgesia for labor: breakthrough or unjustified invasion? Semin Perinatol. 2002;26:109-21. 
Olmez G, Dag IH, Ozyilmaz MA, Yalinkaya A. Can Combined Spinal-Epidural Analgesia be an Alternative to Epidural Analgesia Alone in Labour? Turk J Anaesthesiol Reanim 2003; 31: 66-72.

Poma S, Scudeller L, Verga C, Mirabile G, Gardella B, Broglia $F$ et al. Effects of combined spinalepidural analgesia on first stage of labor: a cohort study. J Matern Fetal Neonatal Med. 2018:1-7. doi: 10.1080/14767058.2018.1467892. [Epub ahead of print]

Sezer AO, Gunaydin B. Efficacy of patient controlled epidural analgesia after initiation with epidural or combined spinal epidural analgesia. Int J Obstet Anesth 2007; 16: 226-30.

Stocks GM, Hallworth SP, Fernando R, England AJ, Columb MO, Lyons G. Minimum local analgesic dose of intrathecal bupivacaine in labor and the effect of intrathecal fentanyl. Anesthesiology 2001; 94: 593-8.

Thomson G, Feeley C, Moran VH, Downe S, Oladapo OT. Women's experiences of pharmacological and non-pharmacological pain relief methods for labour and childbirth: a qualitative systematic review. Reprod Health. 2019 30;16(1):71.

Wong CA. Advances in labor analgesia. Int J Women's Health 2009; 1:139-54.

Wong CA. Epidural and Spinal Analgesia/ Anesthesia for Labor and Delivery. In: Chestnut DH, Polley LS, Tsen LC, Wong CA (eds). Chestnut's Obstetric Anesthesia Principals and Practice. 4th edition. Philadelphia: Elsevier Mosby 2009; 429-92. 